\title{
A Bayesian Approach for Adaptive Multiantenna Sensing in Cognitive Radio Networks
}

\author{
J. Manco-Vásquez, M. Lázaro-Gredilla†, D. Ramírez*, J. Vía and I. Santamaría \\ Dept. of Communications Engineering, University of Cantabria, Spain \\ ${ }^{\dagger}$ Dept. Signal Theory and Commun., Univ. Carlos III de Madrid, Spain \\ *Signal and System Theory Group, University of Paderborn, Germany
}

\begin{abstract}
Much of the recent work on multiantenna spectrum sensing in cognitive radio (CR) networks has been based on generalized likelihood ratio test (GLRT) detectors, which lack the ability to learn from past decisions and to adapt to the continuously changing environment. To overcome this limitation, in this paper we propose a Bayesian detector capable of learning in an efficient way the posterior distributions under both hypotheses. These posteriors summarize, in a compact way, all information seen so far by the cognitive secondary user. Our Bayesian model places priors directly on the spatial covariance matrices under both hypothesis, as well as on the probability of channel occupancy. Specifically, we use inverse-gamma and complex inverse-Wishart distributions as conjugate priors for the null and alternative hypothesis, respectively; and a binomial distribution as the prior for channel occupancy. At each sensing period, Bayesian inference is applied and the posterior for the channel occupancy is thresholded for detection. After a suitable approximation, the posteriors are employed as priors for the next sensing frame, which forms the basis of the proposed Bayesian learning procedure. We also include a forgetting mechanism that allows to operate satisfactorily on time-varying scenarios. The performance of the Bayesian detector is evaluated by simulations and also by means of CR testbed composed of universal radio peripheral (USRP) nodes. Both the simulations and our experimental measurements show that the Bayesian detector outperforms the GLRT in a variety of scenarios.
\end{abstract}

Keywords:

Bayesian Inference, Bayesian Forgetting, Cognitive Radio, Generalized Likelihood Ratio Test (GLRT), Multiantenna Spectrum Sensing.

\section{Introduction}

Cognitive Radio (CR) networks [1],[2],[3] rely on spectrum sensing as a key operation that secondary users (SU) must perform in order to identify whether a wireless communication channel is in use by a licensed primary user (PU) or not [4]. A reliable spectrum sensing stage is crucial to detect spectrum holes that can be subsequently filled with transmissions from SU [5]. To this end, detectors employing multiple antennas have received increased attention recently because they do not require prior knowledge about the PU signalling scheme and are able to work with asynchronously sampled signals $[6,7,8,9,10,11,12]$. These multiantenna detectors 
exploit the fact that under the null hypothesis (only noise) the signals received at the different antennas are spatially uncorrelated, whereas the presence of a PU induces some correlation and/or additional structure in the spatial covariance matrix.

Since the binary hypothesis testing problem involves some unknown parameters (e.g., noise variance and channel), the generalized likelihood ratio test (GLRT) approach has been typically followed to find one-shot detectors in several scenarios [6], [7], [9], [12]. A limitation of GLRT detectors, however, is that they are unable to learn from the environment or to track the smooth changes in the characteristics of the channel or the noise that can be expected between consecutive sensing frames. More precisely, it is reasonable to assume that the time scale of variation of the statistical parameters involved in the detection problem (for instance, noise variance or spacetime PU activity pattern) are much longer than the sensing period. For instance, channel access patterns for primary users have been characterized as slowly time-varying in [13] and more recently in [14]. It is clear that detectors able to learn from past decisions would provide improved performance in these slowly time-varying scenarios. With this goal in mind, in this paper we propose an adaptive Bayesian framework for multiantenna sensing and evaluate its performance both by simulations and by means of a CR testbed.

Bayesian detectors for cognitive radios have been previously proposed in $[15,16,17,18$, 19]. Typically, these works assume a prior distribution for the unknown parameters and apply Bayesian inference to come up with improved parameter estimates and, consequently, more reliable detectors. Nevertheless, they are still one-shot detectors without any learning or adaptation capability. In comparison to these Bayesian approaches, our work presents two main novelties: first, our Bayesian detector places priors directly on the spatial covariance matrices under both hypotheses; and second, it includes learning and forgetting steps that allow to track smooth variations of the channel and noise characteristics.

Specifically, our Bayesian model uses inverse-gamma and complex inverse-Wishart distributions as conjugate priors for the null and alternative hypothesis, respectively; and a binomial distribution as the prior for channel occupancy. The reason for choosing these priors being that under Gaussian noise they are the conjugate priors for this problem and, therefore, the calculations are simplified making exact Bayesian inference tractable. At each sensing period, after applying exact Bayesian inference, the posterior is approximated within the family of the prior to keep the sensing process simple and scalable. Since the posteriors summarize the information gathered so far about the actual CR scenario, they are used as priors for the next sensing period: this represents the learning stage. Furthermore, the procedure is equipped with a forgetting mechanism based on [20] that allows to work on non-stationary environments.

In this paper, we extend some initial results about this Bayesian approach which were presented in [21] and [22]. In particular, we consider the optimal approximation of the posterior according to the Kullback-Leibler (KL) distance, and compare its performance and computational cost with the simple approximation based on thresholding which was discussed in [21]. Also, we present a more in depth study of the proposed CR detector performance for different number of receive antennas, observations and channel conditions. Finally, we also evaluate experimentally the performance of the proposed detector, in comparison to conventional GLRT-based detectors, using to this end a cognitive radio hardware platform based on Universal Software Radio Peripheral (USRP) devices [23].

The rest of the paper is organized as follows. The detection problem for the CR network is formulated in Section II. The Bayesian inference procedure for a single sensing frame is presented in Section III, where two different approximations of the posterior are derived. The learning and forgetting procedure for dealing with multiple sensing frames will be discussed 
in Section IV. In Section V, we analyze the simulation results for different settings including both stationary and non-stationary environments, whereas the results obtained with the CR testbed are presented in Section VI. Finally, our main conclusions are summarized in Section VII.

\section{Preliminaries}

\section{Notation}

In this paper, we use bold-face lower case and bold-face upper case letters for column vectors and matrices, respectively; and light-face lower case letters for scalar quantities. The superscripts $(\bullet)$ and $(\cdot)$ refer to the parameters of the posterior and prior distributions, respectively; and $(\because)$ is used for estimated matrices and scalars. The determinant of a matrix $\mathbf{A}$ is denoted as $|\mathbf{A}|$, its trace as trace $(\mathbf{A})$, the operator $\operatorname{diag}(\mathbf{A})$ refers to a diagonal matrix formed with the elements along the main diagonal of $\mathbf{A},[\mathbf{A}]_{i j}$ denotes the $i j$ element of the matrix, and the superscript $(\cdot)^{H}$ denotes Hermitian. Finally, $\mathbf{x} \sim \mathcal{C N}(\boldsymbol{\mu}, \mathbf{R})$ indicates that $\mathbf{x}$ is a complex circular Gaussian random vector of mean $\boldsymbol{\mu}$ and covariance matrix $\mathbf{R}$.

\subsection{Problem Statement and GLRT detectors}

We consider a cognitive receiver equipped with $L$ antennas that wants to detect whether the channel is occupied by a primary user or not. During the $t$-th sensing frame, the cognitive receiver acquires $n=1, \ldots, N$ snapshots denoted by $\mathbf{x}_{t}[n] \in \mathbb{C}^{L}$. The signal received during the whole sensing period is stacked in a matrix: $\mathbf{X}_{t}=\left[\mathbf{x}_{t}[1], \ldots, \mathbf{x}_{t}[N]\right]$. The spectrum sensing problem can be formulated as a binary hypothesis test as follows

$$
\begin{aligned}
& \mathcal{H}_{1}: \mathbf{x}_{t}[n]=\mathbf{H}_{t} \mathbf{s}_{t}[n]+\mathbf{v}_{t}[n], \\
& \mathcal{H}_{0}: \mathbf{x}_{t}[n]=\mathbf{v}_{t}[n],
\end{aligned}
$$

where $\mathbf{x}_{t}[n]$ is the acquired snapshot at time $n, \mathbf{s}_{t}[n] \in \mathbb{C}^{P}$ is the primary signal vector, which might represent the signal emitted by a single PU with $P$ antennas or the signals emitted concurrently by $P$ single-antenna PUs (see Fig. 1), $\mathbf{H}_{t} \in \mathbb{C}^{L \times P}$ describes the multiple-input multipleoutput (MIMO) channel between the PU and the cognitive receiver, and $\mathbf{v}_{t}[n]$ is modeled as zeromean additive white Gaussian cicular noise. The distributions of the vector-valued observations under each hypothesis $\mathcal{H}_{1}$ and $\mathcal{H}_{0}$, follow a $\mathcal{C N}\left(\mathbf{0}, \mathbf{R}_{t}\right)$ and $\mathcal{C N}\left(\mathbf{0}, \mathbf{D}_{t}\right)$, respectively. Therefore, without any additional prior knowledge about the modulation format or signalling scheme used by the PU, the spectrum sensing problem amounts to testing between two different structures for the covariance matrix of $\mathbf{x}_{t}[n]$ :

$$
\begin{aligned}
& \mathcal{H}_{1}: \mathbf{x}_{t}[n] \sim \mathcal{C N}\left(\mathbf{0}, \mathbf{R}_{t}\right), n=0, \ldots, N-1 . \\
& \mathcal{H}_{0}: \mathbf{x}_{t}[n] \sim \mathcal{C N}\left(\mathbf{0}, \mathbf{D}_{t}\right), n=0, \ldots, N-1 .
\end{aligned}
$$

where we assume that $\mathbf{R}_{t}$ is an arbitrary positive definite covariance matrix and $\mathbf{D}_{t}$ is a diagonal covariance matrix.

Notice also that the likelihood under each hypothesis depends on unknown parameters and therefore the hypotheses are composite. The most typical approach to solve this kind of testing problems is the generalized likelihood ratio test (GLRT). When the noise is independent and 


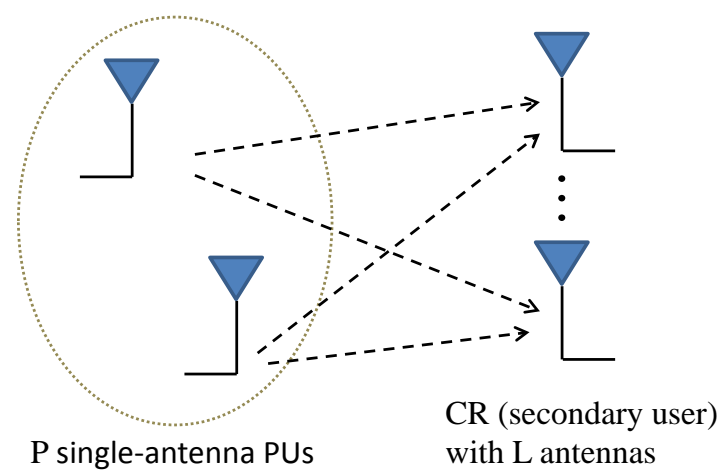

Figure 1: CR detection model: a cognitive user with $L$ antennas tries to detect the presence of $P \geq 1$ single-antenna PUs or, equivalently, a single $P$-antenna PU.

identically distributed (iid) at each antenna $\left(\mathbf{D}_{t}=\sigma^{2} \mathbf{I}\right)$ and $P \geq L-1$, the GLRT is the wellknown sphericity test [24], ${ }^{1}$ which is given by

$$
\mathscr{L}_{S}=\frac{\left|\mathbf{S}_{t}\right|^{1 / L}}{(1 / L) \operatorname{trace}\left(\mathbf{S}_{t}\right)}
$$

where $\mathbf{S}_{t}=\mathbf{X}_{t} \mathbf{X}_{t}^{H} / N$ is the sample covariance matrix.

A more general testing problem that can accommodate calibration uncertainties in the different antenna front-ends, takes into account a generic diagonal noise covariance matrix under $\mathcal{H}_{0}$. This GLRT in this case is the Hadamard ratio [25] and is given by

$$
\mathscr{L}_{H}=\frac{\left|\mathbf{S}_{t}\right|}{\prod_{i=1}^{L}\left[\mathbf{S}_{t}\right]_{i i}} .
$$

\section{Bayesian inference on a single sensing frame}

The Bayesian approach proposed in this paper assigns prior distributions to the covariance matrices under both hypotheses, as well as to the probability of channel occupancy. After discussing which priors should be used for this problem, in this section we perform exact Bayesian inference over a single sensing frame to derive the posteriors for the unknown parameters. Specifically, the posterior for the channel occupancy is the statistic used to decide whether the SU should transmit or not.

\subsection{Prior distributions}

Let us first introduce $z_{t}$ as a binary hidden random variable that indicates whether a transmitter is present $\left(z_{t}=1\right)$ or not $\left(z_{t}=0\right)$. Let us also remind that all our information about $\mathbf{R}_{t}$ and $\mathbf{D}_{t}$ is that they are some unknown covariance matrices, respectively full Hermitian and diagonal.

\footnotetext{
${ }^{1}$ For rank-deficient signal covariance matrices the GLRT is, in general, more complicated, as it was shown in [9].
} 
Following a proper Bayesian treatment, prior distributions on all the unknown parameters of the model $\left(z_{t}, \mathbf{R}_{t}\right.$ and $\left.\mathbf{D}_{t}\right)$ must be placed. We will use the following:

$$
\begin{aligned}
p\left(z_{t}\right) & =\operatorname{Bernoulli}\left(z_{t} \mid \breve{\pi}_{t}\right)=\breve{\pi}_{t}^{z_{t}}\left(1-\breve{\pi}_{t}\right)^{1-z_{t}} \\
p\left(\mathbf{R}_{t}\right) & =C \mathcal{W}^{-1}\left(\mathbf{R}_{t} \mid \breve{n}_{t}, \breve{\mathbf{R}}_{t}\right) \\
& =\frac{\left|\breve{\mathbf{R}}_{t}\right|^{\frac{\breve{t}_{t}}{2}}\left|\mathbf{R}_{t}\right|^{-\frac{\breve{n}_{t}+L+1}{2}} \exp \left(-\frac{1}{2} \operatorname{trace}\left(\mathbf{R}_{t}^{-1} \breve{\mathbf{R}}_{t}\right)\right)}{2^{\frac{\breve{h}_{t} L}{2}} \Gamma_{L}\left(\frac{\breve{n}_{t}}{2}\right)} \\
p\left(\mathbf{D}_{t}\right) & =\mathcal{G}_{L}^{-1}\left(\mathbf{D}_{t} \mid \breve{m}_{t}, \breve{\mathbf{D}}_{t}\right) \\
& =\prod_{l=1}^{L} \mathcal{G}^{-1}\left(\left[\mathbf{D}_{t}\right]_{l l} \mid \breve{m}_{t} / 2,\left[\breve{\mathbf{D}}_{t}\right]_{l l} / 2\right) \\
& =\frac{\left|\breve{\mathbf{D}}_{t}\right|^{\frac{\breve{m}_{t}}{2}}\left|\mathbf{D}_{t}\right|^{-\frac{\breve{m}_{t}+L+1}{2}} \exp \left(-\frac{1}{2} \operatorname{trace}\left(\mathbf{D}_{t}^{-1} \breve{\mathbf{D}}_{t}\right)\right)}{2^{\frac{\breve{t}_{t} L}{2}} \Gamma^{L}\left(\frac{\breve{m}_{t}}{2}\right)}
\end{aligned}
$$

where we have included the definitions of the Bernoulli distribution, the complex inverse-Wishart $\left(C W^{-1}\right)$ and the product of $L$ independent inverse-gamma $\left(\mathcal{G}_{L}^{-1}\right)$. Note the difference between $\Gamma_{L}(\cdot)$ (used to denote the multivariate gamma function) and $\Gamma^{L}(\cdot)$ (the standard gamma function raised to the $L$-th power). We denote the parameters of the prior distributions as $\breve{\pi}_{t}, \breve{n}_{t}, \breve{\mathbf{R}}_{t}, \breve{m}_{t}$ and $\breve{\mathbf{D}}_{t}$. When the SU starts sensing the environment (i.e., at $t=0$ ), the prior parameters should be as uninformative as possible. They will be learnt over time as new sensing frames are acquired according to the mechanism that will be described in Section 4.

The main argument for the choice of these priors is analytical tractability: The complex inverse-Wishart distribution placed on $\mathbf{R}_{t}$, and the product of univariate inverse-gamma distributions placed on $\mathbf{D}_{t}$ are the conjugate priors for the distribution of full-rank covariance matrices and diagonal covariance matrices, respectively, when the observations follow a complex multivariate Gaussian distribution. As we will see in the next subsection, these conjugate priors allow us to exactly perform a Bayesian inference which is very convenient to avoid resorting to numerical integration methods.

\subsection{Exact posterior distribution of $z_{t}, \mathbf{R}_{t}$ and $\mathbf{D}_{t}$}

Since the noise is assumed to be Gaussian, the likelihood of $\mathbf{R}_{t}$ and $\mathbf{D}_{t}$ given $\mathbf{X}_{t}$ can be written as

$$
\begin{aligned}
& p\left(\mathbf{X}_{t} \mid z_{t}=0, \mathbf{D}_{t}\right)=\prod_{n=1}^{N} \mathcal{C N}\left(\mathbf{x}[n] \mid z_{t}=0, \mathbf{D}_{t}\right), \\
& p\left(\mathbf{X}_{t} \mid z_{t}=1, \mathbf{R}_{t}\right)=\prod_{n=1}^{N} \mathcal{C N}\left(\mathbf{x}[n] \mid z_{t}=1, \mathbf{R}_{t}\right) .
\end{aligned}
$$

Given the hidden variable, $z_{t}$, priors are conjugate and therefore posterior distributions have the same form as the prior, but with different parameters. When $z_{t}$ is marginalized, each posterior is a convex combination of the posteriors for each hypothesis, yielding

$$
\begin{aligned}
p\left(z_{t} \mid \mathbf{X}_{t}\right) & =\operatorname{Bernoulli}\left(z_{t} \mid \hat{\pi}_{t}\right) \\
p\left(\mathbf{R}_{t} \mid \mathbf{X}_{t}\right) & =\hat{\pi}_{t} C \mathcal{W}^{-1}\left(\mathbf{R}_{t} \mid \hat{n}_{t}, \hat{\mathbf{R}}_{t}\right)+\left(1-\hat{\pi}_{t}\right) C \mathcal{W}^{-1}\left(\mathbf{R}_{t} \mid \breve{n}_{t}, \breve{\mathbf{R}}_{t}\right) \\
p\left(\mathbf{D}_{t} \mid \mathbf{X}_{t}\right) & =\hat{\pi}_{t} \mathcal{G}_{L}^{-1}\left(\mathbf{D}_{t} \mid \breve{m}_{t}, \breve{\mathbf{D}}_{t}\right)+\left(1-\hat{\pi}_{t}\right) \mathcal{G}_{L}^{-1}\left(\mathbf{D}_{t} \mid \hat{m}_{t}, \hat{\mathbf{D}}_{t}\right) .
\end{aligned}
$$


The posterior parameters are given by

$$
\begin{aligned}
\hat{n}_{t} & =\breve{n}_{t}+N \\
\hat{\mathbf{R}}_{t} & =\breve{\mathbf{R}}_{t}+\mathbf{S}_{t} \\
\hat{m}_{t} & =\breve{m}_{t}+N \\
\hat{\mathbf{D}}_{t} & =\breve{\mathbf{D}}_{t}+\operatorname{diag}\left(\mathbf{S}_{t}\right) \\
\hat{\pi}_{t} & =\frac{p\left(\mathbf{X}_{t} \mid z_{t}=1\right) p\left(z_{t}=1\right)}{p\left(\mathbf{X}_{t} \mid z_{t}=1\right) p\left(z_{t}=1\right)+p\left(\mathbf{X}_{t} \mid z_{t}=0\right) p\left(z_{t}=0\right)}
\end{aligned}
$$

Recall that we use a breve $(\checkmark)$ to denote the parameters of the prior distribution, whereas we use a hat $\left({ }^{\wedge}\right)$ to denote the parameters of the posterior distribution. Finally, the marginal likelihood $p\left(\mathbf{X}_{t} \mid z_{t}\right)$ can be obtained analytically as

$$
\begin{aligned}
p\left(\mathbf{X}_{t} \mid z_{t}=1\right) & =\int p\left(\mathbf{X}_{t} \mid z_{t}=1, \mathbf{R}_{t}\right) p\left(\mathbf{R}_{t}\right) \mathrm{d} \mathbf{R} \\
& =\frac{\left|\breve{\mathbf{R}}_{t}\right|^{\frac{\breve{n}_{t}}{2}} \Gamma_{L}\left(\frac{\hat{n}_{t}}{2}\right)}{\pi^{\frac{N L}{2}}\left|\hat{\mathbf{R}}_{t}\right|^{\frac{\hat{n}_{t}}{2}} \Gamma_{L}\left(\frac{\breve{n}_{t}}{2}\right)} \\
p\left(\mathbf{X}_{t} \mid z_{t}=0\right) & =\int p\left(\mathbf{X}_{t} \mid z_{t}=0, \mathbf{D}_{t}\right) p\left(\mathbf{D}_{t}\right) \mathrm{d} \mathbf{D} \\
& =\frac{\left|\breve{\mathbf{D}}_{t}\right|^{\frac{\breve{m}_{t}}{2}} \Gamma\left(\frac{\hat{m}_{t}}{2}\right)^{L}}{\pi^{\frac{N L}{2}}\left|\hat{\mathbf{D}}_{t}\right|^{\frac{\hat{m}_{t}}{2}} \Gamma\left(\frac{\breve{m}_{t}}{2}\right)^{L}} .
\end{aligned}
$$

After the posterior has been computed, the probability of a transmitter being present given observations $\mathbf{X}_{t}$ is simply $p\left(z_{t}=1 \mid \mathbf{X}_{t}\right)=\hat{\pi}_{t}$. Thus, we can occupy the channel when the collision probability $\hat{\pi}_{t}$ is below some desired threshold.

\section{Bayesian inference over multiple frames}

\subsection{Learning from past sensing frames}

The posteriors after processing the $t$-th frame summarizes all statistical information observed so far. Therefore, a natural learning mechanism is to use them as priors for the next sensing frame, as depicted in Fig. 2. More specifically, the proposed learning procedure is as follows: at each sensing frame the cognitive receiver updates the posterior distribution for $\mathbf{R}_{t}$ and $\mathbf{D}_{t}$ from priors existing at $t$ and the likelihood obtained from $\mathbf{X}_{t}$; then, these posteriors become the priors to be used at the sensing period $t+1$. The procedure is started with uninformative priors at $t=0$.

A problem with a direct application of this idea is that, after applying Bayesian inference, the posterior distributions for $\mathbf{R}_{t}$ and $\mathbf{D}_{t}$ are convex combinations of the posteriors under each hypotheses, see Eqs. (7b) and (7c); and therefore the posterior does not belong to the same family distribution of the prior. For instance, the prior for $\mathbf{R}_{t}$ is a complex inverse-Wishart and the posterior is a linear combination of two complex inverse-Wisharts. To keep the process simple and scalable, it would be convenient to find an approximation of the posteriors within the family of each respective prior. In the next subsections we describe two possible approximations that can be applied to this end. 


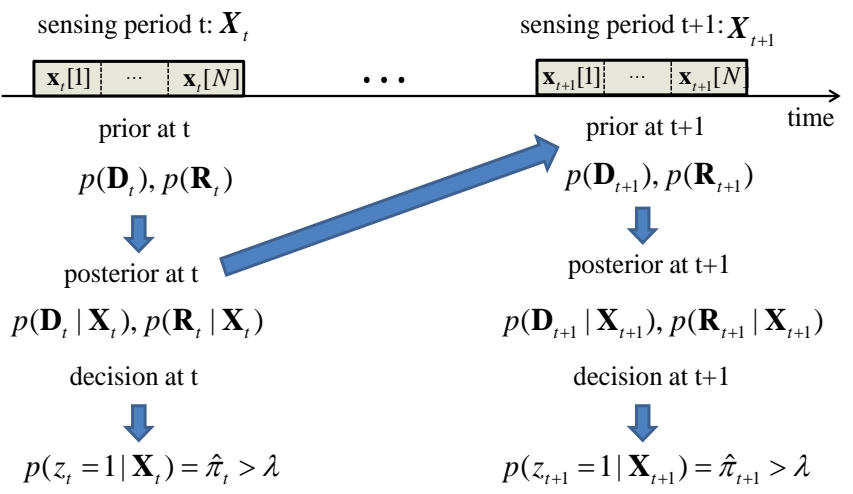

Figure 2: A Bayesian framework for spectrum sensing: the posteriors obtained after processing a sensing frame are employed as priors for the next sensing frame.

\subsubsection{Thresholding-based approximation}

A simple approximation to the posterior that falls within the same family as the prior can be obtained by truncating $\hat{\pi}_{t}$ to either 0 or 1 , whichever it is closer. When this is done, Eq. (7b) and $(7 \mathrm{c})$ directly yields a posterior in the same family as the prior. In that case, when $\mathcal{H}_{1}$ is more probable, the posterior is obtained by performing only updates (8a) and (8b), whereas in the opposite case, only updates $(8 \mathrm{c})$ and $(8 \mathrm{~d})$ are needed.

\subsubsection{Kullback-Leibler approximation}

A more rigorous approach is to find the approximation of the posteriors within the family of the priors that minimize the Kullback-Leibler distance. More precisely, the exact posteriors (reproduced here for convenience) are given by

$$
\begin{aligned}
& p\left(\mathbf{R}_{t} \mid \mathbf{X}_{t}\right)=\hat{\pi}_{t} C \mathcal{W}^{-1}\left(\mathbf{R}_{t} \mid \hat{n}_{t}, \hat{\mathbf{R}}_{t}\right)+\left(1-\hat{\pi}_{t}\right) C \mathcal{W}^{-1}\left(\mathbf{R}_{t} \mid \breve{n}_{t}, \breve{\mathbf{R}}_{t}\right), \\
& p\left(\mathbf{D}_{t} \mid \mathbf{X}_{t}\right)=\hat{\pi}_{t} \mathcal{G}_{L}^{-1}\left(\mathbf{D}_{t} \mid \breve{m}_{t}, \breve{\mathbf{D}}_{t}\right)+\left(1-\hat{\pi}_{t}\right) \mathcal{G}_{L}^{-1}\left(\mathbf{D}_{t} \mid \hat{m}_{t}, \hat{\mathbf{D}}_{t}\right) .
\end{aligned}
$$

Our problem consists in finding the approximations of these posteriors

$$
\begin{aligned}
& q\left(\mathbf{R}_{t} \mid \mathbf{X}_{t}\right)=C \mathcal{W}^{-1}\left(\mathbf{R}_{t} \mid \tilde{n}_{t}, \tilde{\mathbf{R}}_{t}\right), \\
& q\left(\mathbf{D}_{t} \mid \mathbf{X}_{t}\right)=\mathcal{G}_{L}^{-1}\left(\mathbf{D}_{t} \mid \tilde{m}_{t}, \tilde{\mathbf{D}}_{t}\right),
\end{aligned}
$$

that minimize the Kullback-Leibler (KL) divergence. Therefore, we have to solve the following optimization problems

$$
\begin{aligned}
\left\{\tilde{m}_{t}, \tilde{\mathbf{D}}_{t}\right\} & =\underset{\tilde{m}_{t}, \tilde{\mathbf{D}}_{t}}{\operatorname{argmin}} \operatorname{KL}\left(p\left(\mathbf{D}_{t} \mid \mathbf{X}_{t}\right) \| q\left(\mathbf{D}_{t} \mid \mathbf{X}_{t}\right)\right) \\
\left\{\tilde{n}_{t}, \tilde{\mathbf{R}}_{t}\right\} & =\underset{\tilde{n}_{t}, \tilde{\mathbf{R}}_{t}}{\operatorname{argmin}} \operatorname{KL}\left(p\left(\mathbf{R}_{t} \mid \mathbf{X}_{t}\right) \| q\left(\mathbf{R}_{t} \mid \mathbf{X}_{t}\right)\right) .
\end{aligned}
$$

Fortunately, each of these minimization problems can be solved analytically except for a line search. The details of the derivation are relegated to Appendix A, in the following we 


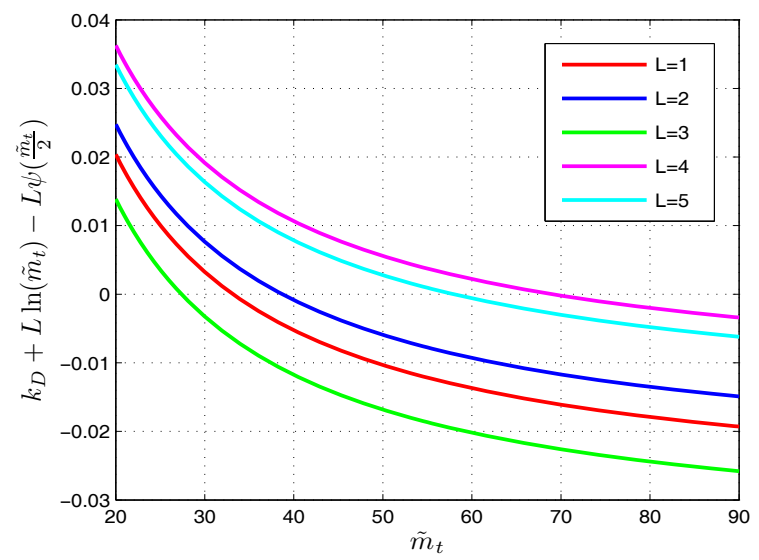

Figure 3: The value of the function $k_{D}+L \ln \left(\tilde{m}_{t}\right)-L \psi\left(\frac{\tilde{m}_{t}}{2}\right)$ versus $\tilde{m}_{t}$ for different number of antennas $L$.

summarize the solution. In order to find $\left\{\tilde{m}_{t}, \tilde{\mathbf{D}}_{t}\right\}$ and $\left\{\tilde{n}_{t}, \tilde{\mathbf{R}}_{t}\right\}$, we first compute the following auxiliary quantities

$$
\begin{aligned}
\mathbf{K}_{D}= & \hat{\pi}_{t} \breve{m}_{t} \breve{\mathbf{D}}_{t}^{-1}+\left(1-\hat{\pi}_{t}\right) \hat{m}_{t} \hat{\mathbf{D}}_{t}^{-1} \\
\mathbf{K}_{R}= & \hat{\pi}_{t} \hat{n}_{t} \hat{\mathbf{R}}_{t}^{-1}+\left(1-\hat{\pi}_{t}\right) \breve{n}_{t} \breve{\mathbf{R}}_{t}^{-1} \\
k_{D}= & -\ln \left|\mathbf{K}_{D}\right|+\hat{\pi}_{t}\left(L \psi\left(\frac{\breve{m}_{t}}{2}\right)-\ln \left|\breve{\mathbf{D}}_{t}\right|\right) \\
& +(1-\hat{\pi})\left(L \psi\left(\frac{\hat{m}_{t}}{2}\right)-\ln \left|\hat{\mathbf{D}}_{t}\right|\right) \\
k_{R}= & -\ln \left|\mathbf{K}_{R}\right|+\hat{\pi}_{t}\left(\psi_{L}\left(\frac{\hat{n}_{t}}{2}\right)-\ln \left|\hat{\mathbf{R}}_{t}\right|\right) \\
& +(1-\hat{\pi})\left(\psi_{L}\left(\frac{\breve{n}_{t}}{2}\right)-\ln \left|\breve{\mathbf{R}}_{t}\right|\right)
\end{aligned}
$$

where $\psi(\cdot)$ is the digamma function, and $\psi_{L}(\cdot)=\sum_{l=1}^{L} \psi(\cdot+(1-l) / 2)$ defines the multivariate digamma function.

We then have to solve the following non-linear equations using, for instance, a few iterations of the Newton-Raphson method

$$
\begin{aligned}
k_{D}+L \ln \left(\tilde{m}_{t}\right)-L \psi\left(\frac{\tilde{m}_{t}}{2}\right) & =0, \\
k_{R}+L \ln \left(\tilde{n}_{t}\right)-\psi_{L}\left(\frac{\tilde{n}_{t}}{2}\right) & =0 .
\end{aligned}
$$

As an example, Fig. 3 depicts the nonlinear equation (14a) for different values of $L$. In all cases the function is smooth and its zero can be easily found. Finally, the covariance matrices $\tilde{\mathbf{D}}_{t}$ and $\tilde{\mathbf{R}}_{t}$ for the best approximation according to the KL distance are given by $\tilde{m}_{t} \mathbf{K}_{\mathbf{D}}^{-1}$ and $\tilde{n}_{t} \mathbf{K}_{\mathbf{R}}^{-1}$, respectively. These values are taken as the new parameters of the posterior distributions, that is 


$$
\begin{aligned}
\tilde{\mathbf{D}}_{t} & \rightarrow \hat{\mathbf{D}}_{t}, \\
\tilde{\mathbf{R}}_{t} & \rightarrow \hat{\mathbf{R}}_{t}, \\
\tilde{n}_{t} & \rightarrow \hat{n}_{t}, \\
\tilde{n}_{t} & \rightarrow \hat{m}_{t} .
\end{aligned}
$$

\subsection{Forgetting in non-stationary environments}

Since the channel may vary between consecutive frames, it is interesting to introduce a mechanism within the Bayesian framework to forget past data and hence be able to operate in a nonstationary environment. We assume here that no additional knowledge about the dynamical evolution of the channel, PU spectrum usage pattern or noise statistics is available. Therefore, we resort to the idea of Bayesian $\lambda$-forgetting [20] that allows to forget in a principled Bayesian manner with minimal assumptions. The basic idea of Bayesian forgetting is to use as prior distributions for frame $t+1$ a "smoothed" version of the posterior distributions obtained after processing frame $t$ and the original prior distributions for $\mathbf{R}_{t}$ and $\mathbf{D}_{t}$ given by (5), i.e.,

$$
\begin{aligned}
& p\left(\mathbf{D}_{t+1} \mid \mathbf{X}_{t}\right) \propto p\left(\mathbf{D}_{t} \mid \mathbf{X}_{t}\right)^{\lambda} p\left(\mathbf{D}_{0}\right)^{1-\lambda,}, \\
& p\left(\mathbf{R}_{t+1} \mid \mathbf{X}_{t}\right) \propto p\left(\mathbf{R}_{t} \mid \mathbf{X}_{t}\right)^{\lambda} p\left(\mathbf{R}_{0}\right)^{1-\lambda} .
\end{aligned}
$$

Observe that according to this definition, when $\lambda=0$, all the information obtained from previous data is forgotten and the process considers each frame independently (as the GLRT does), which is reasonable if abrupt changes occur in $\mathbf{R}_{t}$ and $\mathbf{D}_{t}$ between frames. When $\lambda=1$, no forgetting occurs and the new posterior corresponds to the standard Bayesian posterior when $\mathbf{D}_{t}$ and $\mathbf{R}_{t}$ are constant across frames $\mathbf{D}_{t}=\mathbf{D}, \mathbf{R}_{t}=\mathbf{R} \forall t$, which is reasonable under stationary conditions. Values of $\lambda \in[0,1]$ are therefore appropriate to model different evolution speeds in the channel, without having to define a concrete dynamical model. In another perspective, Eqs. (16) represent a change of the posterior in the direction of the prior: this has also been named as "back-to-theprior" forgetting in [26].

With this forgetting step, the parameters of the prior distributions to be used for Bayesian inference at $t+1$ are given by

$$
\begin{aligned}
\breve{n}_{t+1} & =\lambda \hat{n}_{t}+(1-\lambda) \breve{n}_{0} \\
\breve{\mathbf{R}}_{t+1} & =\lambda \hat{\mathbf{R}}_{t}+(1-\lambda) \breve{\mathbf{R}}_{0} \\
\breve{m}_{t+1} & =\lambda \hat{m}_{t}+(1-\lambda) \breve{m}_{0} \\
\breve{\mathbf{D}}_{t+1} & =\lambda \hat{\mathbf{D}}_{t}+(1-\lambda) \breve{\mathbf{D}}_{0} .
\end{aligned}
$$

\subsection{The proposed algorithm}

The whole process is summarized in Algorithm 1. Since the algorithm only requires updating and storing $\hat{\mathbf{R}}_{t}, \hat{n}_{t}, \hat{\mathbf{D}}_{t}, \hat{m}_{t}$ from one frame to the next, it requires a fixed amount of memory and computation per sensing frame, which is $O\left(L^{2}\right)$. 


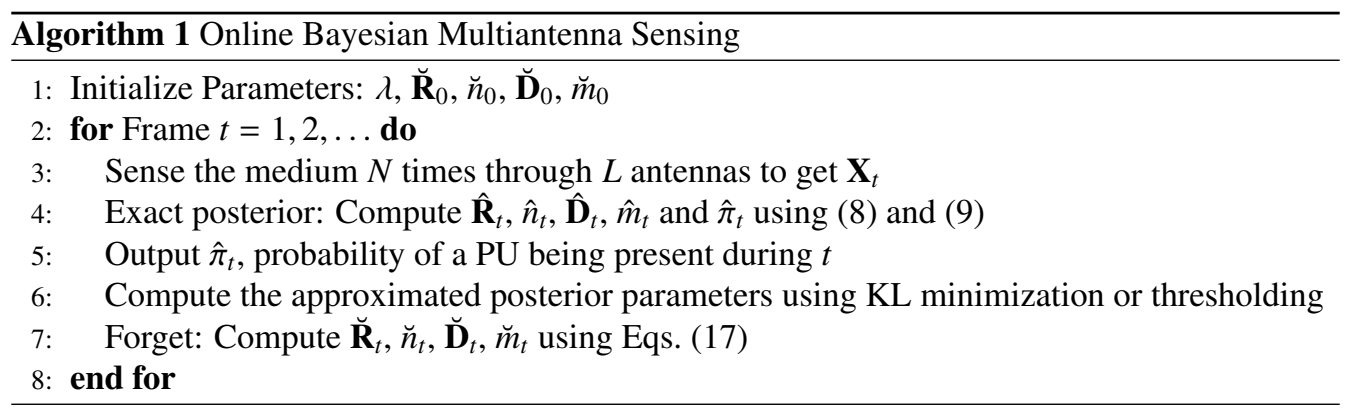

\section{Simulation results}

In this section, we compare the performance of the proposed Bayesian detector with that of a GLRT-based detector (given by (4)) in different environments by means of Monte Carlo simulations. Unless otherwise stated, we assume a probability of channel occupancy given by $\breve{\pi}_{t}=0.5$, a primary transmitter with $P=5$ antennas and a secondary cognitive receiver with $L=5$ antennas. The MIMO channel matrix is assumed to be constant during the $t$-th sensing frame with i.i.d. entries distributed as $C \mathcal{N}(0,1)$. On the other hand, the channel evolves from frame to frame as $\mathbf{H}_{t+1}=\lambda_{c h} \mathbf{H}_{t}+\left(1-\lambda_{c h}\right) \mathbf{P}_{t+1}$ [27], with $0 \leq \lambda_{c h} \leq 1$, and $\mathbf{P}_{t+1}$ a complex Gaussian noise matrix also with i.i.d. entries distributed as $C \mathcal{N}(0,1)$. For $\lambda_{c h}=1$ we have a stationary channel, whereas for $\lambda_{c h}=0$ it changes independently from frame to frame according to a block-fading model.

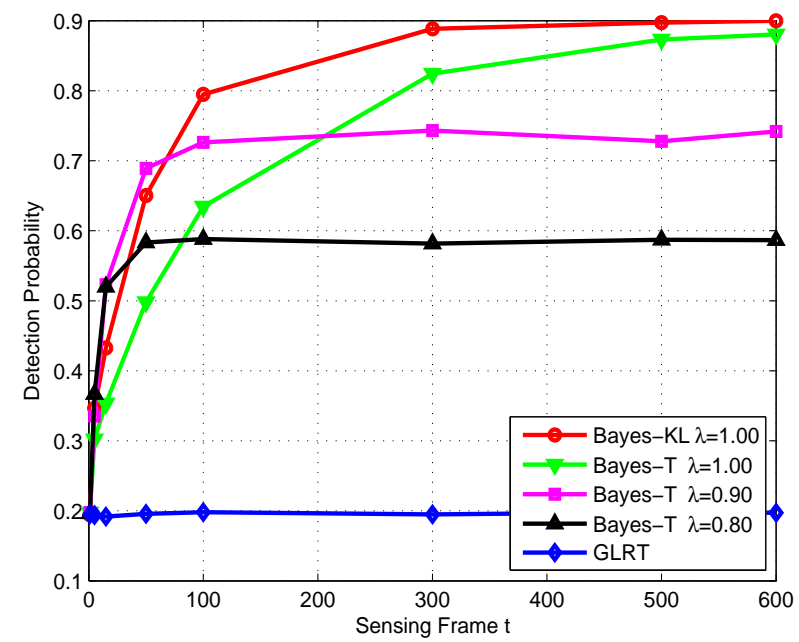

Figure 4: $P_{D}$ for the Bayesian detector (Bayes-KL and Bayes-T) and the GLRT vs. the number of sensing frames in a time-invariant channel, $L=5, P=5, N=50, \mathrm{SNR}=-8 \mathrm{~dB}$ and $P_{F A}=0.1$. 


\section{1. $P_{D}$ versus number of sensing frames}

In this subsection, we study how the performance of the Bayesian detector evolves over time in stationary, slowly time-varying and fast time-varying environments. We start at $t=0$ with an uninformative prior and then after each sensing frame we update the posterior (learning step), approximate the posterior using either truncation (denoted as Bayes- $\mathrm{T}$ in the plots) or KL minimization (denoted as Bayes-KL) and finally forget moving the approximated posterior towards the original uninformative prior with a forgetting factor $\lambda$. As a figure of merit we plot the detection probability $P_{D}$ versus the number of sensing frames. We consider a fixed false alarm probability of $P_{F A}=0.1$, and in each sensing frame the number of observations is $N=50$. For comparison we include the results obtained with the GLRT. In all examples we use a a signal-to-noise ratio $\mathrm{SNR}=-8 \mathrm{~dB}$.

\subsubsection{Stationary channel}

We first consider a static scenario for which the channel remains constant over all sensing frames (i.e., $\lambda_{c h}=1$ ). The results in Fig. 4 show that in this scenario, after just a few sensing frames, the Bayesian multiantenna detector provides a much higher $P_{D}$ than the GLRT for different values of the forgetting parameter $\lambda$. After observing a sufficient number of frames the best results are obtained when using $\lambda=1$ (which means no forgetting at all), as could be expected for this static environment. Interestingly, however, to forget a little $(\lambda=0.9)$ can be beneficial during the first sensing frames. This is explained because during the first sensing frames detection errors are more likely to occur and, consequently, the parameters of the posterior are not updated correctly. In this situation, it would be better not to trust so much on the observed data and apply the forgetting step. Finally, we also compare in the figure the performance of the two approximations of the posterior proposed in the paper. As expected, the KL-based approximation provides a better performance at the cost of a higher computational complexity.

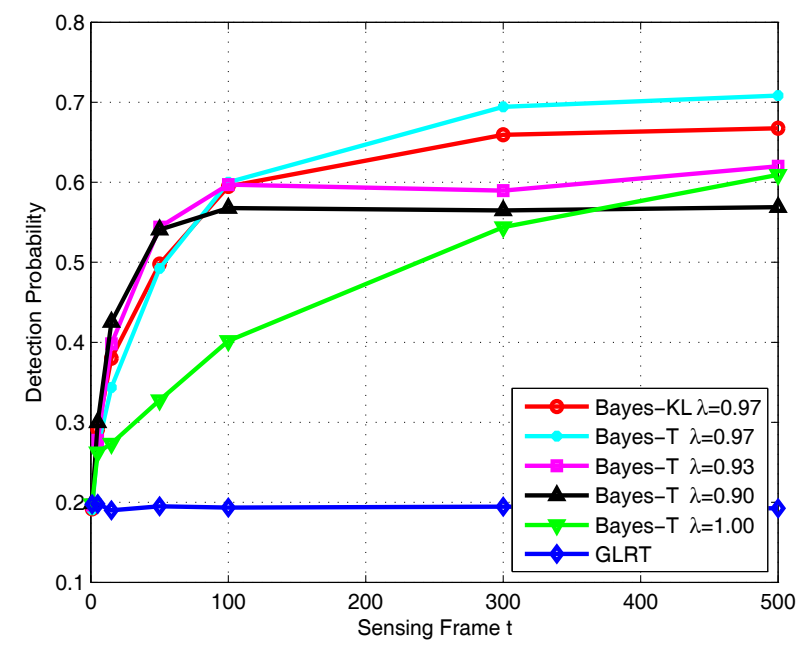

Figure 5: $P_{D}$ for the Bayesian detector (Bayes-KL and Bayes-T) and the GLRT vs. the number of sensing frames in a slowly time-invariant channel. $L=5, P=5, N=50, \mathrm{SNR}=-8 \mathrm{~dB}, P_{F A}=0.1$ and $\lambda_{c h}=0.9$. 


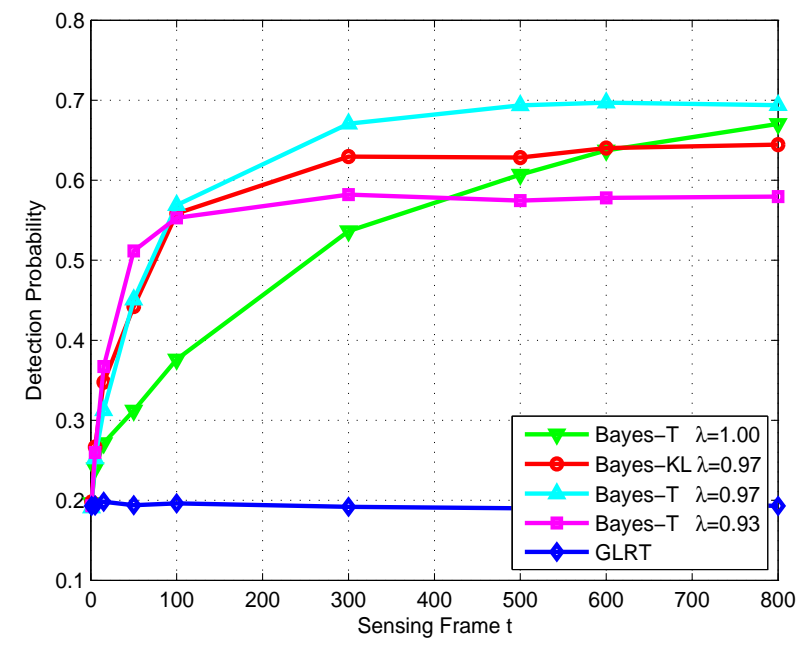

Figure 6: $P_{D}$ for the Bayesian detector (Bayes-KL and Bayes-T) and the GLRT vs. the number of sensing frames in a fast time-varying channel. $L=5, P=5, N=50, \mathrm{SNR}=-8 \mathrm{~dB}, P_{F A}=0.1$, and $\lambda_{c h}=0.1$.

\subsubsection{Slowly time-varying channel}

We now consider a non-stationary environment created by a slowly time-varying channel with $\lambda_{c h}=0.9$. The results in Fig. 5 show again that the Bayesian detector outperforms the GLRT after just a few sensing frames. The optimal value of the forgetting factor for this scenario seems to be close to $\lambda=0.97,{ }^{2}$ and using a value of $\lambda=1$ (no forgetting) strongly affects the performance. It is also clear that the convergence now is slower, since it takes more sensing frames to effectively learn and track the covariance matrices under both hypotheses. Finally, regarding the impact of the posterior approximation on the performance of the detector, we observe that in non-stationary environments it is better to use the coarse approximation of the posterior obtained by truncation. In non-stationary environments, the importance of obtaining at each step an accurate approximation diminishes since the performance is limited by the variations observed from frame to frame.

\subsubsection{Fast time-varying channel}

In Fig. 6, we finally consider the case of a fast time-varying environment with $\lambda_{c h}=0.1$. Remarkably, even in this highly non-stationary environment, the Bayesian detector outperforms the GLRT detector. This improvement can be attributed to the fact that the covariance matrix under $\mathcal{H}_{0}$ remains almost constant from frame to frame (only the channel changes) and, therefore, it can be learnt by the Bayesian detector. This improved estimate of the noise-only covariance matrix translates into a better $P_{D}$ in comparison to the GLRT. For the reasons explained before, the simple truncation of the posterior performs better than the most accurate KL-based approximation in this rapidly varying scenario.

\footnotetext{
${ }^{2}$ Let us point out that this value has no direct relationship with $\lambda_{c h}$.
} 


\subsection{Receiver Operating Characteristic}

In this subsection, we obtain the receiver operating characteristic (ROC) curve of the detector after convergence, i.e., after processing a sufficient number of frames to reach the steady state. We study the ROC curve for different number of observations per sensing frame $(N=$ $\{50,30,15\})$. We also concentrate on the results provided by the approximation of the posterior referred to as Bayes-T; since, as we haven seen previously, the approximation based on the KL distance is computationally more costly and does not show any improvement in time-varying environments.

Fig.7(a) shows the results for the stationary channel, with $\lambda=1$ and SNR $=-8 \mathrm{~dB}$. As we see, in steady-state the proposed Bayesian detector with only 15 snapshots per sensing frame outperforms the GLRT with 50 snapshots, which means a reduction of more than three times in the sensing time per frame. Fig. 7(b) and Fig. 7(c) show the results for slowly (using $\lambda_{c h}=$ 0.90 ) and fast (using $\lambda_{c h}=0.10$ ) time-varying environments, respectively; from which similar conclusions can be drawn.

Finally, we fix the detection threshold and evaluate the probability of detection, $P_{D}$, and false alarm, $P_{F A}$, for different SNRs and in different scenarios. The number of samples per sensing frame is fixed to $N=50$ and the rest of parameters is the same as in the previous section. The results are shown in Fig. 8(a), Fig. 8(b) and Fig. 8(c) for stationary, slowly and fast time-varying scenarios.

\subsection{Detection performance for a rank-P $P U$}

In Fig. 9, we compare the probability of missed detection, $P_{M}$, for the Bayesian and GLRT detectors when the spatial rank of the PU signal varies. For the GLRT detector we have used the results in [9]. We consider an scenario with $L=6, N=50, P_{F A}=0.1, P=\{1, \ldots, 6\}$ and $\mathrm{SNR}=-8 \mathrm{~dB}$. In general, the performance of both detectors degrade for an increasing $P$, since as $P$ increases the covariance matrix under $\mathcal{H}_{1}$ has less structure to be exploited. Nevertheless, the Bayesian approach consistently provides better results than the GLRT, which validates again its ability to learn from the environment even when the actual model does not match exactly the presumed one.

\section{Experimental Evaluation}

In this section, we further validate the simulations by means of experimental measurements on a low-cost hardware cognitive platform. Specifically, our platform is composed of several N210 Universal Software Radio Peripheral (USRP) devices [23], each of them consists of a USRP motherboard and a Radio Frequency (RF) daughterboard (the XCVR2450 daughterboard based on a MAX2829 IC is able to cover ISM bands of $2.4 \mathrm{GHz}$ to $2.5 \mathrm{GHz}$, and $4.9 \mathrm{GHz}$ to $5.8 \mathrm{GHz}$ ). Basically, the motherboard consists of dual analog-to-digital converters (ADC) and digital-to-analog (DAC) converters connected to a Field Programmable Gain Array (FPGA). On the other hand, the daughterboard is a modular front-end used for analog operations such as up/down conversion.

In order to implement a multiantenna cognitive node, the N210 USRP includes a specific expansion port that allows coherent synchronization of two USRP2 units, as it is depicted in Fig. 10. Since the same clock (oscillators) and time reference are shared, both USRP nodes can start transmitting/receiving at the same time, thus avoiding any synchronization problem. 


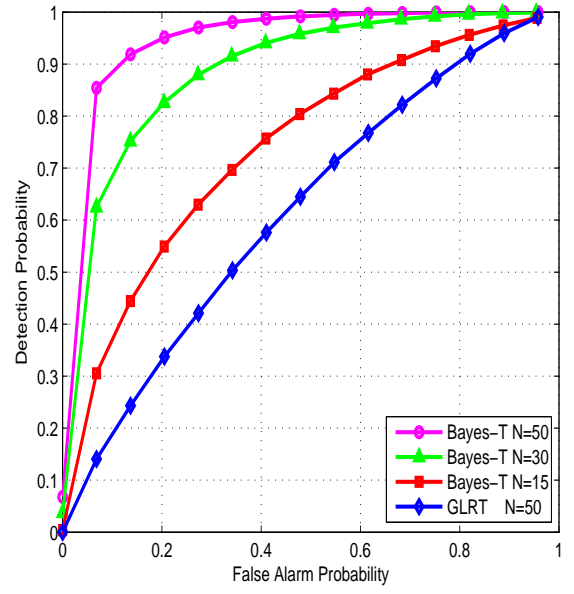

(a)

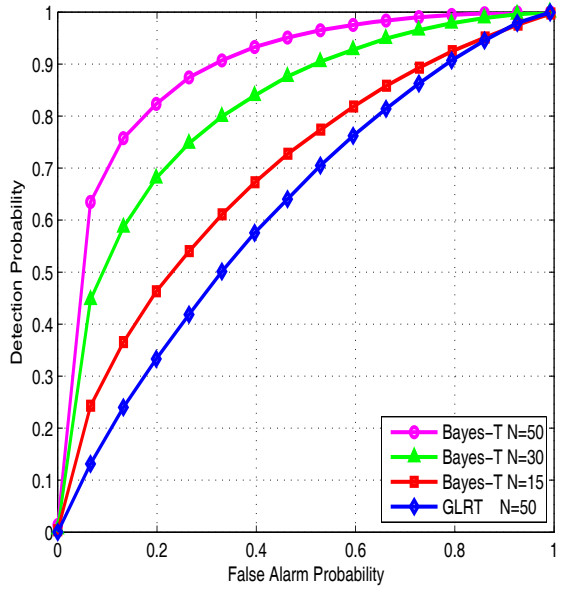

(b)

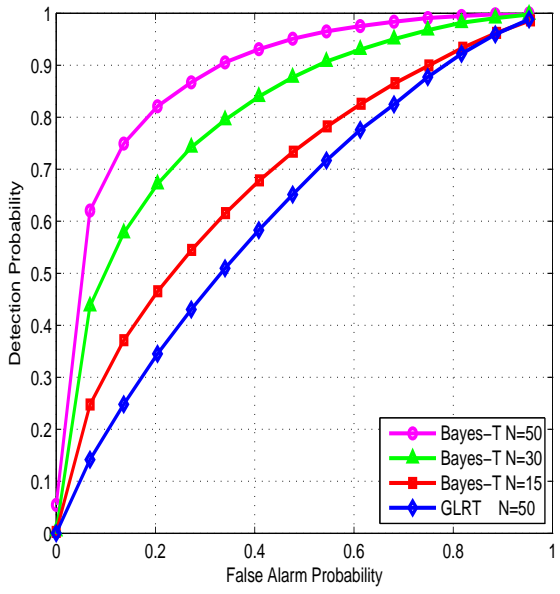

(c)

Figure 7: ROC curves for the Bayesian and GLRT detector with $L=5, P=5$ and different number of snapshots per sensing frame. (a) Stationary channel with SNR $=-8 \mathrm{~dB}$ and $\lambda=1.0$. (b) Slowly time-varying channel with $\mathrm{SNR}=-8 \mathrm{~dB}, \lambda=0.97$ and $\lambda_{c h}=0.90$. (c) Fast time-varying channel with $\mathrm{SNR}=-8 \mathrm{~dB}, \lambda=0.97$ and $\lambda_{c h}=0.10$ 


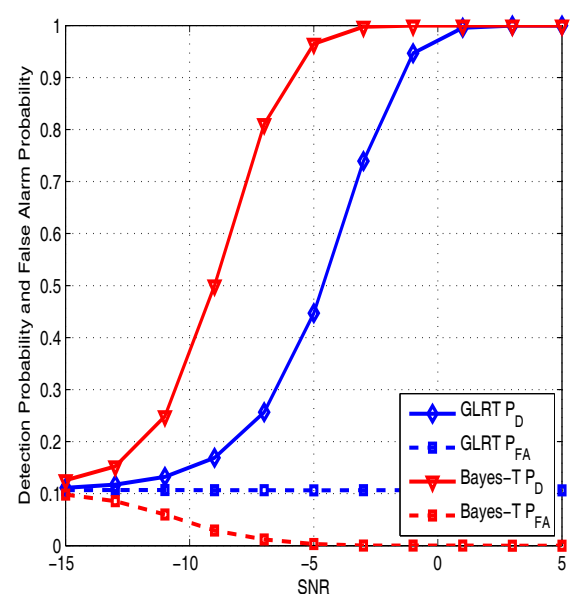

(a)

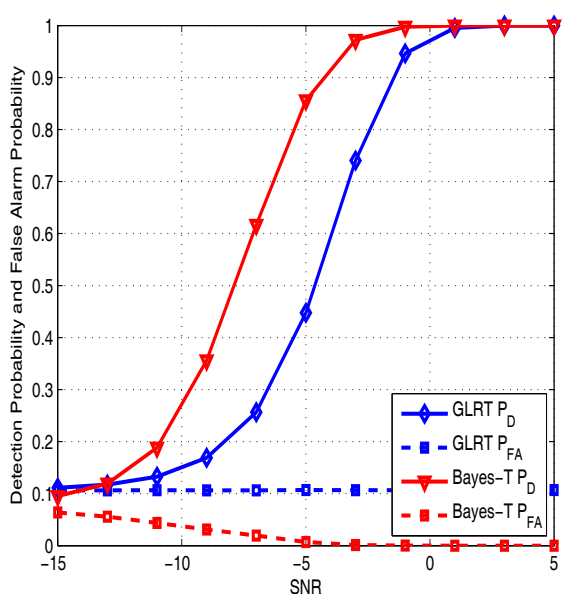

(b)

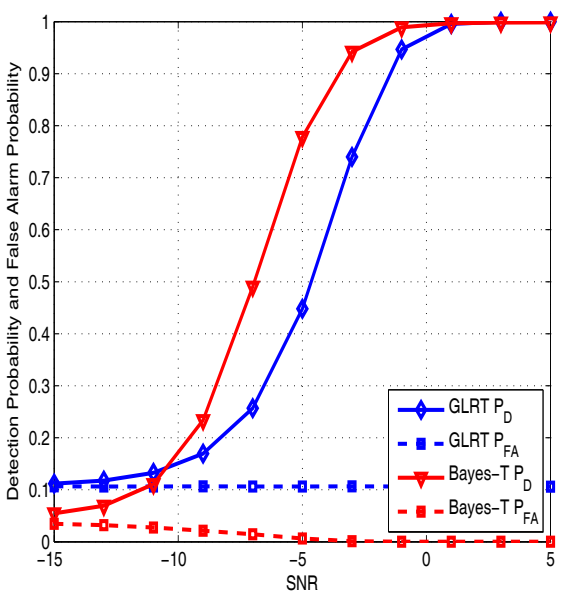

(c)

Figure 8: $P_{D}$ and $P_{F A}$ vs SNR with $L=5$ antennas, and $N=50$. (a) In a time-invariant channel $\left(\lambda=1\right.$ and $\left.\lambda_{c h}=1\right)$. (b) In a slowly time-varying channel, $\left(\lambda=0.97\right.$ and $\lambda_{c h}=0.95$. (c) In a fast time-varying channel $\left(\lambda=0.95\right.$ and $\left.\lambda_{c h}=0.10\right)$. 


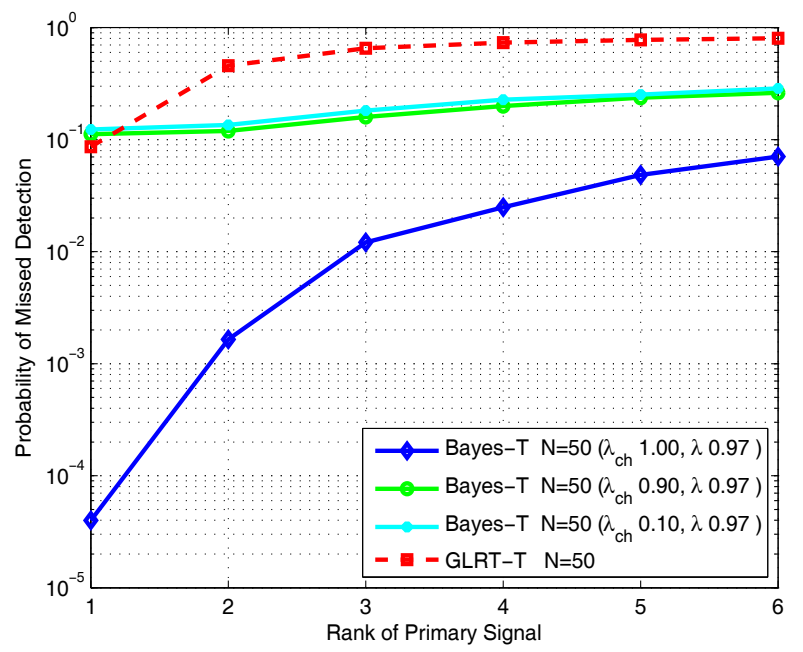

Figure 9: Probability of missed detection versus the rank of the primary signal $P$ for both detectors with $L=6$ antennas, $N=50, \mathrm{SNR}=-8 \mathrm{~dB}$ and $P_{F A}=0.1$. A static time-invariant channel with $\lambda=1.0 \mathrm{using}$ a KL posterior approximation, a slowly time-varying channel with $\lambda=0.97$ and $\lambda_{c h}=0.90$, and a fast time-varying channel with $\lambda=0.97$ and $\lambda_{c h}=0.10$.

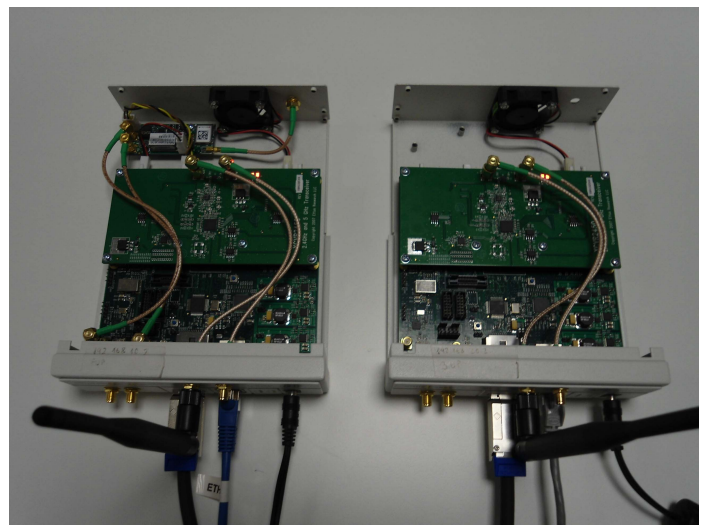

Figure 10: N210 Ettus devices with the XCVR2450 daugtherboard installed. A two-antenna cognitive receiver is composed of two N210 boards connected through a MIMO cable. 
We have considered a simple scenario where a single-antenna PU access the channel according to a predefined pattern and a cognitive receiver with two antennas senses periodically the medium and applies different detection procedures. The platform is controlled from a central PC, which allows us to define a pattern of spectrum occupancy as well as the sensing periods (see Fig. 11). Therefore, at each sensing period we know exactly the true hypothesis and hence we can estimate $P_{D}$ and $P_{F A}$ for given threshold.

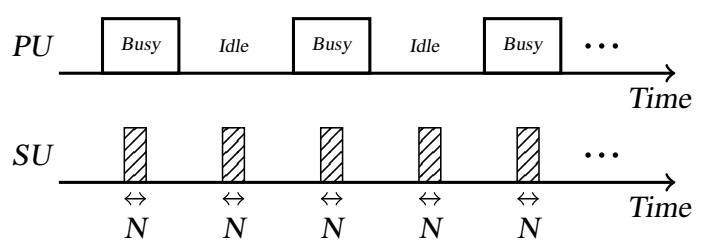

Figure 11: A PU transmits according to a preestablished sequence of states, and the SU senses periodically the wireless channel.

The PU transmits an orthogonal frequency division multiplexing (OFDM) 802.11a signal at a carrier frequency of $5.6 \mathrm{GHz}$, although during the detection stage the modulation format is assumed to be unknown by the SU. The experiments were conducted in the laboratory of the Signal Processing Group at the University of Cantabria, with a clear line of sight (LOS) between the PU and the cognitive receiver in a rather static environment. For each experiment, the SNR is controlled by the transmitter power and measured from the received signal at baseband. For the example shown in this section the measured SNR was $-7.3 \mathrm{~dB}$, the number of samples acquired by the SU during each sensing frame was $N=50$, the number of SU antennas is $L=2$ and the number of PU antennas is $P=1$. In Fig. 12, we compare the ROC obtained by the proposed Bayesian detector working with a forgetting factor of $\lambda=0.99$ and the GLRT detector for this setup. This figures corroborates the validity of the simulations carried out in Section 5. For a full detailed description of the experimental evaluation, the reader is referred to [22] and [28], where a procedure to emulate time-varying scenarios is also described.

\section{Conclusion}

We have derived a new Bayesian framework for the problem of multiantenna spectrum sensing. We assume that the observations follow a Gaussian distribution under both hypotheses, which allows us to choose conjugate priors and thereby to perform the exact Bayesian inference with closed-form expressions. Moreover, our Bayesian framework is able to exploit previous statistical information obtained from past sensing frames. To that end, we propose a forgetting mechanism where the posterior densities on the covariance matrices summarize this past information and the next Bayesian inference takes these posteriors as suitable priors. We evaluate the derived Bayesian framework in different scenarios, that is, stationary and non-stationary environments. The comparison between the Bayesian framework and GLRT detector under these scenarios as well as experimental evaluations shows that the Bayesian detector outperforms the GLRT. The most interesting findings are provided under a time-varying environment, where we showed that the Bayesian detector is able to efficiently learn the posterior.

\section{ACKNOWLEDGMENT}




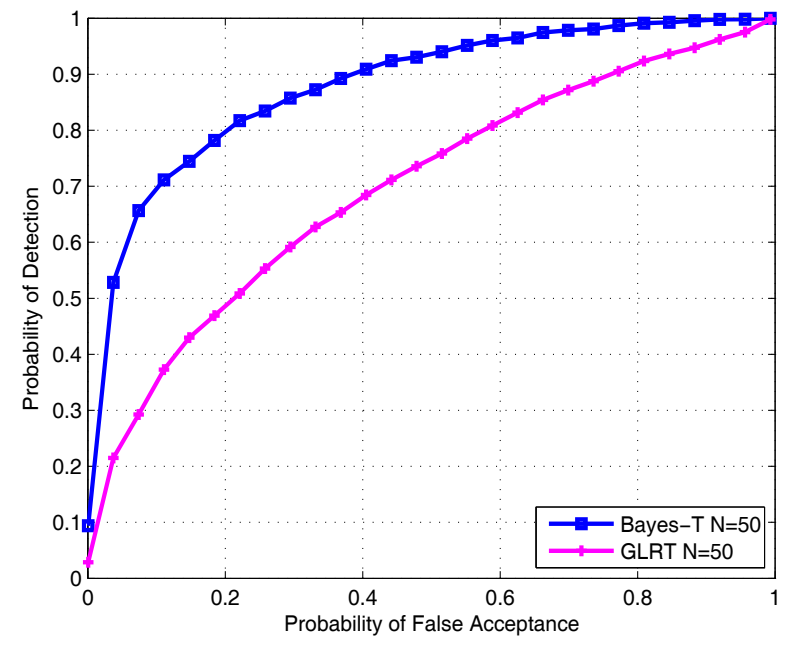

Figure 12: ROC curves for the Bayesian and GLRT detectors using the CR platform in a realistic indoor channel at 5,6 GHz.

The research leading to these results has received funding from the Spanish Government (MICINN) under projects TEC2010-19545-C04-03 (COSIMA) and CONSOLIDER-INGENIO 2010 CSD200800010 (COMONSENS). It also has been supported by FPI grant BES-2011-047647.

\section{Appendix A. Derivation of KL approximation}

In this appendix we find the pdf approximation that is closest in terms of the KL distance to the exact posterior. For notational simplicity, we omit the subindex $t$ which refers to the sensing frame. We will only consider the approximation under $\mathcal{H}_{1}$, since the derivations under the null hypothesis are exactly the same. More precisely, under $\mathcal{H}_{1}$ the exact posterior is given by

$$
p(\mathbf{R} \mid \mathbf{X})=\hat{\pi} C \mathcal{W}^{-1}(\mathbf{R} \mid \hat{n}, \hat{\mathbf{R}})+(1-\hat{\pi}) C \mathcal{W}^{-1}(\mathbf{R} \mid \breve{n}, \breve{\mathbf{R}}),
$$

and we want to find the approximation

$$
q(\mathbf{R} \mid \mathbf{X})=C \mathcal{W}^{-1}(\mathbf{R} \mid \tilde{n}, \tilde{\mathbf{R}}),
$$

that minimizes the KL divergence. Than is, we want to solve

$$
\{\tilde{n}, \tilde{\mathbf{R}}\}=\underset{\tilde{n}, \tilde{\mathbf{R}}}{\operatorname{argmin}} \operatorname{KL}(p(\mathbf{R} \mid \mathbf{X}) \| q(\mathbf{R} \mid \mathbf{X}))
$$

where,

$$
\mathrm{KL}(p(\mathbf{R} \mid \mathbf{X}) \| q(\mathbf{R} \mid \mathbf{X}))=\int p(\mathbf{R} \mid \mathbf{X}) \ln (p(\mathbf{R} \mid \mathbf{X})) \mathrm{d} \mathbf{R}-\int p(\mathbf{R} \mid \mathbf{X}) \ln (q(\mathbf{R} \mid \mathbf{X})) \mathrm{d} \mathbf{R}
$$


The first term in the right hand side of (A.4) is the negative of the differential entropy of the exact posterior and hence does not depend on $\{\tilde{n}, \tilde{\mathbf{R}}\}$. Therefore, minimizing the $\mathrm{KL}$ is equivalent to solving the following maximization problem

$$
\{\tilde{n}, \tilde{\mathbf{R}}\}=\underset{\tilde{n}, \tilde{\mathbf{R}}}{\operatorname{argmax}} \int p(\mathbf{R} \mid \mathbf{X}) \ln (q(\mathbf{R} \mid \mathbf{X})) \mathrm{d} \mathbf{R} .
$$

Now, by substituting (A.1) and (A.2) into (A.5), we have

$$
\begin{aligned}
\int p(\mathbf{R} \mid \mathbf{X}) \ln (q(\mathbf{R} \mid \mathbf{X})) \mathrm{d} \mathbf{R}= & \int \hat{\pi} \ln \left(C \mathcal{W}^{-1}(\mathbf{R} \mid \tilde{n}, \tilde{\mathbf{R}})\right) C \mathcal{W}^{-1}(\mathbf{R} \mid \hat{n}, \hat{\mathbf{R}}) \mathrm{d} \mathbf{R} . \\
& +\int(1-\hat{\pi}) \ln \left(C \mathcal{W}^{-1}(\mathbf{R} \mid \tilde{n}, \tilde{\mathbf{R}})\right) C \mathcal{W}^{-1}(\mathbf{R} \mid \breve{n}, \breve{\mathbf{R}}) \mathrm{d} \mathbf{R} .
\end{aligned}
$$

Integrating the first term on the right-hand side of Eq. (A.6) we get

$$
\begin{aligned}
& \int \hat{\pi} \ln \left(C \mathcal{W}^{-1}(\mathbf{R} \mid \tilde{n}, \tilde{\mathbf{R}})\right) C \mathcal{W}^{-1}(\mathbf{R} \mid \hat{n}, \hat{\mathbf{R}}) \mathrm{d} \mathbf{R} \\
& =\hat{\pi}\left[\int \ln \left(\frac{|\tilde{\mathbf{R}}|^{\frac{\tilde{n}}{2}}}{2^{-\frac{\tilde{n}}{2}} \Gamma_{L}\left(\frac{\tilde{n}}{2}\right)}\right) C \mathcal{W}^{-1}(\mathbf{R} \mid \hat{n}, \hat{\mathbf{R}}) \mathrm{d} \mathbf{R}\right]+\hat{\pi}\left[-\frac{\tilde{n}+L+1}{2} \int \ln (|\mathbf{R}|) C \mathcal{W}^{-1}(\mathbf{R} \mid \hat{n}, \hat{\mathbf{R}}) \mathrm{d} \mathbf{R}\right] \\
& +\hat{\pi}\left[-\frac{1}{2} \int \operatorname{trace}\left(\mathbf{R}^{-1} \tilde{\mathbf{R}}\right) C \mathcal{W}^{-1}(\mathbf{R} \mid \hat{n}, \hat{\mathbf{R}}) \mathrm{d} \mathbf{R}\right] \\
& =\hat{\pi}\left[\frac{\tilde{n}}{2} \ln |\tilde{\mathbf{R}}|-\frac{\tilde{n}}{2} L \ln (2)-\ln \Gamma_{L}\left(\frac{\tilde{n}}{2}\right)\right]+\hat{\pi}\left[-\frac{\tilde{n}+L+1}{2}\left(\ln |\hat{\mathbf{R}}|-L \ln (2)-\psi_{L}\left(\frac{\hat{n}}{2}\right)\right)\right]+\hat{\pi}\left[-\frac{1}{2} \operatorname{trace}\left(\hat{n} \hat{\mathbf{R}}^{-1} \tilde{\mathbf{R}}\right)\right]
\end{aligned}
$$

where in the last step, we have used the fact that ${ }^{3} \mathbb{E}[\ln |\mathbf{R}|]=\ln (|\hat{\mathbf{R}}|)-L \ln (2)-\psi_{L}\left(\frac{\hat{n}}{2}\right)$ and $\mathbb{E}\left[\mathbf{R}^{-1}\right]=\hat{n} \hat{\mathbf{R}}^{-1}$. By the same procedure, the second term on the right-hand side of Eq. (A.6) is given by

$$
\begin{aligned}
& \int(1-\hat{\pi}) \ln \left(C \mathcal{W}^{-1}(\mathbf{R} \mid \tilde{n}, \tilde{\mathbf{R}})\right) C \mathcal{W}^{-1}(\mathbf{R} \mid \breve{n}, \breve{\mathbf{R}}) \mathrm{d} \mathbf{R} \\
& =(1-\hat{\pi})\left[\frac{\tilde{n}}{2} \ln |\tilde{\mathbf{R}}|-\frac{\tilde{n}}{2} L \ln 2-\ln \left(\Gamma_{L}\left(\frac{\tilde{n}}{2}\right)\right)\right]+(1-\hat{\pi})\left[-\frac{\tilde{n}+L+1}{2}\left(\ln |\breve{\mathbf{R}}|-L \ln (2)-\psi_{L}\left(\frac{\breve{n}}{2}\right)\right]\right. \\
& +(1-\hat{\pi})\left[-\frac{1}{2} \operatorname{trace}\left(\breve{n} \breve{\mathbf{R}}^{-1} \tilde{\mathbf{R}}\right)\right]
\end{aligned}
$$

Combining the two terms yields $\int p(\mathbf{R} \mid \mathbf{X}) \ln (q(\mathbf{R} \mid \mathbf{X})) \mathrm{d} \mathbf{R}$. In order to obtain the parameters that maximize this function, we have to take derivatives with respect to $\tilde{n}$ and $\tilde{\mathbf{R}}$ and equate them to zero. We first derive $\int p(\mathbf{R} \mid \mathbf{X}) \ln (q(\mathbf{R} \mid \mathbf{X})) \mathrm{d} \mathbf{R}$ with respect to $\tilde{\mathbf{R}}$, that is

$$
\hat{\pi}\left[\frac{\tilde{n}}{2} \tilde{\mathbf{R}}^{-1}-\frac{\hat{n}}{2} \hat{\mathbf{R}}^{-1}\right]+(1-\hat{\pi})\left[-\frac{\tilde{n}}{2} \tilde{\mathbf{R}}^{-1}-\frac{\breve{n}}{2} \breve{\mathbf{R}}^{-1}\right]=0
$$

where we have applied the identities $\partial \ln \left|\boldsymbol{\Sigma}_{\mathbf{1}}\right| / \partial \boldsymbol{\Sigma}_{\mathbf{1}}=\left(\boldsymbol{\Sigma}_{\mathbf{1}}{ }^{T}\right)^{-1}$ and $\partial \operatorname{trace}\left(\boldsymbol{\Sigma}_{\mathbf{2}} \boldsymbol{\Sigma}_{\mathbf{1}}\right) / \partial \boldsymbol{\Sigma}_{\mathbf{1}}=\boldsymbol{\Sigma}_{\mathbf{2}}{ }^{T}$. By defining $\mathbf{K}_{R}=\left(\hat{\pi} \hat{n} \hat{\mathbf{R}}^{-1}+(1-\hat{\pi}) \breve{n} \breve{\mathbf{R}}^{-1}\right)^{-1}$, it readily follows that $\tilde{\mathbf{R}}=\tilde{n} \mathbf{K}_{R}^{-1}$.

\footnotetext{
${ }^{3}$ Recall that $\psi_{L}(a)=\frac{\partial}{\partial a} \ln \left(\Gamma_{L}(a)\right)=\sum_{l=1}^{L} \psi(a+(1-l) / 2)$.
} 
Now we take the derivative of $\int p(\mathbf{R} \mid \mathbf{X}) \ln (q(\mathbf{R} \mid \mathbf{X})) \mathrm{d} \mathbf{R}$ with respect to $\tilde{n}$, which is given by

$$
\begin{gathered}
\hat{\pi}\left[\frac{1}{2} \ln |\tilde{\mathbf{R}}|-\frac{L}{2} \ln (2)-\frac{1}{2} \psi\left(\frac{\breve{n}}{2}\right)\right]-\hat{\pi}\left[\frac{1}{2} \ln |\hat{\mathbf{R}}|-\frac{L}{2} \ln (2)-\frac{1}{2} \psi_{L}\left(\frac{\hat{n}}{2}\right)\right] \\
+(1-\hat{\pi})\left[\frac{1}{2} \ln |\tilde{\mathbf{R}}|-\frac{L}{2} \ln (2)-\frac{1}{2} \psi\left(\frac{\breve{n}}{2}\right)\right]-(1-\hat{\pi})\left[\frac{1}{2} \ln |\breve{\mathbf{R}}|-\frac{L}{2} \ln (2)-\frac{1}{2} \psi_{L}\left(\frac{\breve{n}}{2}\right)\right]=0
\end{gathered}
$$

Finally using $\tilde{\mathbf{R}}=\tilde{n} \mathbf{K}_{R}^{-1}$, and defining $k_{R}=-\ln \left|\mathbf{K}_{R}\right|+\hat{\pi}\left(\psi_{L}\left(\frac{\hat{n}}{2}-\ln |\hat{\mathbf{R}}|\right)+(1-\hat{\pi})\left(\psi_{L}\left(\frac{\breve{n}}{2}-\ln |\breve{\mathbf{R}}|\right)\right.\right.$, the non-linear equation $k_{R}+L \ln \tilde{n}-\psi_{L}\left(\frac{\tilde{n}}{2}\right)=0$ is obtained. The same approach is followed to compute its counterpart under the hypothesis $\mathcal{H}_{0}$.

\section{References}

[1] J. Mitola, G. Q Maguire Jr, Cognitive radio: Making software radios more personal, IEEE Personal Communications 6 (4) (1999) 13-18.

[2] S. Haykin, Cognitive radio: Brain-empowered wireless communications, IEEE Journal on Selected Areas in Communications 23 (2) (2005) 201-220.

[3] I. F. Akyildiz, W.-Y. Lee, M. C. Vuran, S. Mohanty, Next generation dynamic spectrum access cognitive radio wireless networks: A survey, Computer Networks (Elsevier) 50 (13) (2006) 2127-2159.

[4] T. Yucek, H. Arslan, A survey of spectrum sensing algorithms for cognitive radio applications, IEEE Communications Surveys \& Tutorials 11 (1) (2009) 116-130.

[5] S. V. Nagaraj, Entropy-based spectrum sensing in cognitive radio, Signal Processing 89 (2) (2009) 174-180.

[6] P. Wang, J. Fang, N. Han, H. Li, Multiantenna-assisted spectrum sensing for cognitive radio, IEEE Transactions on Vehicular Technology 59 (4) (2010) 1791-1800.

[7] J. Sala-Alvarez, G. Vazquez-Vilar, R. Lopez-Valcarce, Multiantenna GLR detection of rank-one signals with known power spectrum in white noise with unknown spatial correlation, IEEE Transactions on Signal Processing 60 (6) (2012) 3065-3078

[8] J. Tugnait, On multiple antenna spectrum sensing under noise variance uncertainty and flat fading, IEEE Transactions on Signal Processing 60 (4) (2012) 1823-1832.

[9] D. Ramirez, G. Vazquez-Vilar, R. Lopez-Valcarce, J. Via, I. Santamaria, Detection of rank-P signals in cognitive radio networks with uncalibrated multiple antennas, IEEE Transactions on Signal Processing 59 (8) (2011) 3764 3774.

[10] R. Zhang, T. Lim, Y.-C. Liang, Y. Zeng, Multi-antenna based spectrum sensing for cognitive radios: A GLRT approach, IEEE Transactions on Communications 58 (1) (2010) 84-88.

[11] A. Taherpour, M. Nasiri-Kenari, S. Gazor, Multiple antenna spectrum sensing in cognitive radios, IEEE Transactions on Wireless Communications 9 (2) (2010) 814-823.

[12] D. Ramirez, J. Via, I. Santamaria, L. L. Scharf, Detection of spatially correlated Gaussian times series, IEEE Transactions on Signal Processing 58 (10) (2010) 5006-5015.

[13] T. Clancy, B. Walker, Predictive dynamic spectrum access, in: Proc. SDR Forum Technical Conference, 2006.

[14] M. Lopez, Spectrum usage models for the analysis, design and simulation of cognitive radio networks, PhD Thesis, Universitat Politecnica de Catalunya, 2011

[15] J. Font-Segura, X. Wang, GLRT-Based spectrum sensing for cognitive radio with prior information, IEEE Transactions on Communications 58 (7) (2010) 2137-2146.

[16] R. Couillet, M. Debbah, A Bayesian framework for collaborative multi-source signal sensing, IEEE Transactions on Signal Processing 58 (10) (2010) 5186-5195.

[17] E. Axel, E. G. Larsson, A Bayesian approach to spectrum sensing, denoising and anomaly detection, in: Proc. IEEE Int. Conf. on Acoustics, Speech and Signal Processing, 2333-2336, 2009.

[18] R. Couillet, M. Debbah, Bayesian inference for multiple antenna cognitive receivers, in: Proc. IEEE Wireless Communications and Networking Conference, WCNC 2009, 6, 1-6, 2009.

[19] Y. Jin, Cognitive Multi-antenna Radar Detection using Bayesian Inference, in: Proc. of IEEE Sensor Array and Multichannel Signal Processing Workshop (SAM), 2012.

[20] R. Kulhavý, M. B. Zarrop, On general concept of forgetting, International Journal of Control 58 (4) (1993) $905-$ 924.

[21] J. Manco-Vasquez, M. Lazaro-Gredilla, D. Ramirez, J. Via, I. Santamaria, Bayesian multiantenna sensing for cognitive radio, in: Proc. of IEEE Sensor Array and Multichannel Signal Processing Workshop (SAM), 2012. 
[22] J. Manco-Vasquez, J. G. Teran, J. Perez-Arriaga, J. Ibañez, I. Santamaria, Experimental evaluation of multiantenna spectrum sensing detectors using a cognitive radio testbed, in: Proc. of IEEE International Symposium on Signals Systems and Electronics (ISSSE), Potsdam, Germany, 2012.

[23] Ettus Research LLC. Universal Software Radio., URL http://www . ettus . com/, 2012.

[24] J. W. Mauchly, Significance test for sphericity of a normal n-variate distribution, The Annals of Mathematical Statistics 11 (2) (1940) 204-209.

[25] S. S. Wilks, On the independence of k sets of normally distributed statistical variables, The Annals of Mathematical Statistics 3 (3) (1935) 309-326.

[26] S. Van Vaerenbergh, M. Lazaro-Gredilla, I. Santamaria, Kernel recursive least-squares tracker for time-varying regression, IEEE Transactions on Neural Networks and Learning Systems 23 (8) (2012) 1313-1326.

[27] K. Baddour, N. Beaulieu, Autoregressive modeling for fading channel simulation, IEEE Transactions on Wireless Communications 4 (4) (2005) 1650-1662.

[28] J. Gutiérrez, J. Ibañez, J. Perez, Beamforming-based emulation of spatial and temporal correlated MISO channels, in: Proc. of IEEE International Symposium on Signals Systems and Electronics (ISSSE), Potsdam, Germany, 2012. 